\title{
Cuidados dos enfermeiros obstetras com as gestantes no pré- parto em uma maternidade municipal de Teresina-PI
}

Care of obstetric nurses with pregnant pregnant women in a municipal maternity in Teresina-PI Atención de enfermeras obstétricas con mujeres embarazadas en maternidad municipal en Teresina-PI

Lycia Tainá Lima e Silva ORCID :https://orcid.org/0000-0001-7607-272X Centro Universitário Maurício de Nassau. Brasil E-mail: lyciatainalima24@gmail.com

Marina Ribeiro da Fonseca ORCID: https://orcid.org/0000-0002-7456-9270

Faculdade Unidiferencial. Brasil E-mail: marinah.ribeiro@hotmail.com

Rosane da Silva Santana

ORCID: https:// orcid.org/0000-0002-0601-8223 Universidade Federal do Ceará. Brasil E-mail: rosane_santana5@hotmail.com

Francisco Ricardo de Alcântara ORCID: https://orcid.org/0000-0003-4422-2652 Centro Universitário Maurício de Nassau. Brasil E-mail: ricardoalcantara94@hotmail.com

Mariana Libório Dourado Reis ORCID: https:// orcid.org/0000-0002-0456-0908

Unidades Integradas de Pós-Graduação. Brasil E-mail marianaldr@hotmail.com

Ana Cristina Ferreira Pereira ORCID: https://orcid.org/0000-0002-8429-5496 Instituto de Medicina Integral Fernando Figueira. Brasil E-mail: annicris874@gmail.com

Regivane Silva Costa Gonçalves ORCID: https://orcid.org/0000-0001-8812-9981 Centro Universitário Maurício de Nassau. Brasil E-mail: regicosta196@gmail.com

Elizama Costa dos Santos Sousa ORCID: https://orcid.org/0000-0003-3870-5404 Universidade Federal do Piauí. Brasil E-mail: elizama_dossantoscosta@outlook.com

Tatiana Custódio Das Chagas Pires Galvão ORCID: https://orcid.org/0000-0001-9907-0831

Centro Universitário Santo Agostinho. Brasil E-mail: tatis.a @hotmail.com

Cristiana Pacífico Oliveira

ORCID: https://orcid.org/0000-0002-7094-3333 Universidade Federal do Piauí. Brasil E-mail: Cris.enferm@hotmail.com

Miralice Medeiros Ferreira Barbosa

ORCID: https:// orcid.org/0000-0003-4898-2384 Unidades Integradas de Pós-Graduação, Brasil

E-mail: mir.alice@hotmail.com

Maria das Graças Nunes Pereira ORCID: https://orcid.org/0000-0002-0391-2369 Unidades Integradas de Pós-Graduação, Brasil

E-mail: mariinha_np@hotmail.com

Erilayne Thais de Araujo ORCID: https://orcid.org/0000-0002-4196-1233 Centro Universitário Maurício Nassau. Brasil E-mail: erythais12@gmail.com 


\begin{abstract}
Resumo
Para que o parto ocorra de modo natural é necessário que não haja intercorrências ou procedimentos desnecessários. Para evitar complicações, os enfermeiros devem planejar uma assistência humanizada e efetiva com cuidados individualizados voltados para cada situação vivenciada pela mulher durante todas as fases do parto. O estudo tem como objetivo analisar os cuidados dos enfermeiros obstetras com as gestantes no pré-parto em uma maternidade municipal de Teresina-PI. Trata-se de um estudo qualitativo com abordagem descritiva desenvolvido em uma Maternidade pública no município de Teresina-PI nos meses de agosto a outubro de 2018. Participaram da pesquisa oito enfermeiros obstetras em exercício de sua função. Foram incluídos os enfermeiros com especialização em obstetrícia e que assistem diretamente as gestantes no pré-parto e; excluídos apenas os que estavam de férias ou licença durante a realização da pesquisa. Utilizou-se um roteiro semiestruturado para coleta dos dados e, para análise, foi aplicada a técnica de Análise de Conteúdo. Evidenciou-se que os enfermeiros atuam de forma ampla desde a admissão, orientando sobre o processo do trabalho de parto e suas fases, até os cuidados exercidos durante o pré-parto estimulando para que a gestante tenha dilatação rápida e com dor diminuída. É necessário que os enfermeiros exerçam princípios de humanização da assistência, valorizem o acolhimento, a prática de ouvir a paciente e a relação respeitosa entre profissionais/clientes. Espera-se com o estudo contribuir com a expansão da atividade dos enfermeiros obstetras, e na busca da qualidade dos serviços e principalmente com atendimento integral a mulher durante o trabalho de parto. Palavras-chave: Acompanhante; Cuidados de enfermagem; Enfermeiras obstétricas; Humanização da assistência; Parturientes.
\end{abstract}

\begin{abstract}
For birth to occur naturally it is necessary that there are no unnecessary complications or procedures. To avoid complications, nurses should plan a humanized and effective care with individualized care directed to each situation experienced by the woman during all the phases of labor. The study aims to analyze the care of obstetricians with pregnant women in preterm delivery at a maternity hospital in Teresina-PI. This is a qualitative study with a descriptive approach developed in a Public Maternity in the municipality of Teresina-PI from August to October 2018. The study was attended by eight obstetrician nurses in the exercise of their function. Nurses with specialization in obstetrics and who directly assist pregnant women in the prepartum were included; excluding only those who were on vacation or leave while conducting the survey. A semi-structured script was used to collect the data and, for analysis, the Content Analysis technique was applied. It was evidenced that nurses work broadly from admission, guiding the process of labor and its phases, to the care exercised during the pre-delivery stimulating so that the pregnant woman has rapid dilation and with decreased pain. It is necessary that the nurses exercise principles of humanization of care, value the reception, the practice of listening to the patient and the respectful relationship between professionals / clients. The study is expected to contribute to the expansion of obstetrical nurses' activity, and to the quest for quality of services, especially with full care of women during labor.
\end{abstract}

Keywords: Escorts; Nursing care; Obstetric nurses; Humanization of care; Parturients.

\title{
Resumen
}

Para que el nacimiento ocurra de forma natural es necesario que no haya complicaciones o procedimientos innecesarios. Para evitar complicaciones, el enfermero debe planificar una atención humanizada y eficaz con una atención individualizada dirigida a cada situación. experimentado por la mujer durante todas las fases del parto. El estudio tiene como objetivo analizar la atención de los obstetras con gestantes en parto prematuro en una maternidad de Teresina-PI. Se trata de un estudio cualitativo con abordaje descriptivo desarrollado en una Maternidad Pública del municipio de Teresina-PI de agosto a octubre de 2018. El estudio contó con la presencia de ocho enfermeras obstetras en el ejercicio de su función. Se incluyeron enfermeras con especialización en obstetricia y que atienden directamente a la gestante en el preparto; excluyendo solo a aquellos que estaban de vacaciones o se fueron mientras realizaban la encuesta. Se utilizó un guión semiestructurado para la recolección de datos y, para el análisis, se aplicó la técnica de Análisis de Contenido. Se evidenció que las enfermeras trabajan de manera amplia desde el ingreso, orientando el proceso del parto y sus fases, hasta el cuidado ejercido durante el preparto estimulando para que la gestante tenga una dilatación rápida y con disminución del dolor. Es necesario que los enfermeros ejerciten principios de humanización del cuidado, valoren la acogida, la práctica de escuchar al paciente y la relación respetuosa entre profesionales / clientes. Se espera que el estudio contribuya a la expansión de la actividad de las enfermeras obstétricas y a la búsqueda de la calidad de los servicios, especialmente con la atención integral de la mujer durante el parto.

Palabras clave: Escorts; Cuidado de enfermera; Enfermeras obstétricas; Humanización del cuidado; Parturientas.

\section{Introdução}

Desde os primórdios, o processo fisiológico do nascimento natural é considerado uma experiência única, sendo compartilhado entre familiares de acordo com a cultura e a religião de cada região do país. O parto era realizado por parteiras em ambientes que produziam segurança e conforto para as gestantes, como o próprio lar. As cuidadoras eram tratadas como 
integrantes de confiança da família. Nas décadas seguintes, surgiram novas técnicas médicas para realização do parto (Mattos, Vandenberghe \& Martins, 2016).

O parto passou a ser realizado por meio de técnicas cirúrgicas com sutura e drenagem. Surgiu então o termo de obstetrícia, cuja função básica, seria promover a realização do parto pela prática cirúrgica. O fórcipe obstétrico apresentou-se como instrumento influenciador no consentimento da obstetrícia como uma área tecnológica e científica, e a partir do uso da prática, foi aliado o conceito de que o parto era perigoso para a mulher e o bebê, o que confirmava através das taxas de morbimortalidade materno-infantil, e a presença de um médico era imprescindível, desencadeando um conflito entre médicos e parteiras (Pieszak, et al., 2013).

A medicina passou a explorar as técnicas cirúrgicas como prática exclusiva para o parto, esquecendo-se do processo natural da mulher, permitindo que as particularidades da gestação e do parto fossem colocadas em segundo plano (Santos \& Monteiro, 2017).

Foi nesse cenário de compreensão que a enfermagem na obstetrícia surgiu, resgatando em sua essência de exercício profissional e histórico, a arte do cuidado, respeitando o processo fisiológico e resgatando o cuidado humanizado durante o nascer (Santos, Sousa, Almeida \& Gusmão, 2012).

Em 1998 foi reconhecido no Brasil oficialmente o cuidado do enfermeiro durante o parto, em hospitais conveniados com o Sistema Único de Saúde (SUS). No ano seguinte foram criados, os Centros de Parto Normal (CPN), que permitem assistência ao parto de baixo risco fora dos hospitais, e tem o funcionamento apenas de enfermeiros obstetras responsáveis por todos os cuidados oferecidos as puérperas e aos RNs (Rezende, Soares \& Silva, 2015).

Mais adiante, com a elevação do índice de mortalidade materna, foi criada a Rede Cegonha em 2011, com a responsabilidade de garantir atenção humanizada na gravidez, no parto, puerpério e no direito ao nascimento seguro da criança. Os enfermeiros obstetras passam então, a ser responsáveis pela melhoria na assistência às gestantes antes, durante e após o parto, e são habilitados para avaliar as condições de saúde da mulher e o recém-nascido (Oliveira, Barbosa \& Melo, 2016).

As ações dos enfermeiros estão voltadas para detecção precoce e avaliação das intercorrências que podem surgir, buscando soluções. Os enfermeiros obstetras devem promover um modelo de assistência centrado na mulher, no parto e nascimento garantindo a integralidade, conforme a resolução do COFEN nº 0516, 24 de junho de 2016 (Gomes \& Moura, 2012).

O momento do parto é de extrema importância não só na vida de uma mulher, mas também para os familiares envolvidos. Caracteriza-se como um momento de grande intensidade emocional, marco no caminho da vida, que afeta profundamente as mulheres, os bebês, as famílias, com efeitos importantes e persistentes sobre a sociedade (Gomes, Sousa Pontes, Pereira, Brasil \& Moraes, 2014).

Para que o parto ocorra de modo natural, é necessário que sejam implementadas ações para evitar quaisquer intercorrências ou procedimentos desnecessários. É necessário que sejam adotadas medidas que permitam que o parto seja de fato realizado com métodos humanizados, com assistência holística, garantindo a dignidade da mulher e do bebê. As ações dos enfermeiros devem percorrer todos aspectos e procedimentos técnicos com cuidados humanizados para parturiente e sua família (Silva, Nascimento \& Coelho, 2015).

A enfermagem obstétrica nesse contexto busca inserir os princípios de cuidado e estímulo a físiologia do parir, impulsionando o protagonismo feminino, para que esse momento possa ser vivenciado com autonomia das mulheres. Essa autonomia garante a essas pacientes agir de maneira soberana em relação a si mesma (Pereira \& Bento, 2011).

É necessário que antes do parto, os enfermeiros obstetras conversem com as gestantes, expliquem todo o processo, principalmente para aqueles que nunca tiveram filho e consigam fazer que as parturientes tenham confiança e fiquem menos apreensivas. As orientações são essenciais para as mudanças no comportamento das mulheres e para o nascimento rápido do 
bebê (Frigo et al, 2013).

Os enfermeiros obstetras são responsável pelos exercícios que as gestantes devem fazer no pré-parto como movimentação do quadril, uso de posições confortáveis, deambulação, banho no chuveiro e de imersão, massagens, uso da bola suíça com agachamentos e adoção de posições como o decúbito lateral para melhorar o padrão respiratório (Gayeski \& Bruggemann, 2010).

A pesquisa teve como objetivo analisar os cuidados dos enfermeiros obstetras com as gestantes no pré-parto em uma maternidade municipal de Teresina-PI.

\section{Metodologia}

Trata-se de um estudo qualitativo com abordagem descritiva. A pesquisa foi desenvolvida no Centro Obstétrico de uma Maternidade pública no município de Teresina-PI. A maternidade apresenta no quadro de profissionais de enfermagem nove enfermeiros obstetras, porém para o estudo apenas oito participaram, pois um estava de licença maternidade. Utilizou-se como critérios de inclusão, os enfermeiros com especialização em obstetrícia que atuam na assistência direta com as gestantes no pré-parto e, os que estavam de férias ou licenças durante a realização da pesquisa.

A coleta dos dados ocorreu após a autorização da Comissão de Ética da Fundação Municipal de Saúde de Teresina e aprovação do Comitê de Ética em Pesquisa da Faculdade de Ciências e Tecnologia do Maranhão (FACEMA) com o parecer n ${ }^{\circ}$ 2.887.590. (ANEXO)

Utilizou-se um roteiro semiestruturado contendo informações sobre a caracterização do grupo entrevistado e perguntas abertas com intuito de responder os objetivos do estudo. As entrevistas foram agendadas via telefone e realizadas nas dependências da maternidade, em uma sala privada. Aqueles que aceitaram participar do estudo assinaram o Termo de Consentimento de Livre e Esclarecido (TCLE). As entrevistas foram gravadas com recurso em um aparelho MP4® para garantir a autenticidade dos depoimentos.

Como forma de segurança aos entrevistados, a ética que rege a pesquisa sobre o sigilo e o anonimato, foi utilizado as iniciais ENF em caixa alta, seguido da numeração de 01 a 08, a qual foi determinada a partir da ordem de realização das entrevistas, de modo que todos os sujeitos da pesquisa puderam certificar-se de que suas identidades permaneceriam anônimas.

Para análise dos discursos dos participantes foi utilizada a técnica de "Análise de Conteúdo" que segundo Bardin (2016), trata-se de um conjunto de técnicas de análises das comunicações. Em seguida, os dados seguiram as seguintes etapas: pré-análise, leitura flutuante, a partir da qual pôde-se analisar e conhecer o texto deixando ser invadido por impressões e orientações, de forma a identificar as categorias discursivas; exploração do material, com leituras exaustivas dos conteúdos para organização e sistematização dos discursos, permitindo o agrupamento em categorias; análises dos conteúdos das falas dos sujeitos, reunidas por categorias, etapa na qual identificou-se unidades de significados; tratamento dos resultados, com os discursos analisados foram interpretados de maneira crítica e reflexiva, possibilitando a descrição dos resultados e dos significados que consolidaram o tema.

\section{Resultados e Discussão}

\section{Caracterização dos sujeitos}

Participaram da pesquisa oito enfermeiros obstetras, dos quais sete eram do sexo feminino e um masculino. Cinco dos entrevistados possuem faixa etária entre

21 a 30 anos, três entre 31 a 40 anos. Cinco concluíram a graduação em enfermagem em instituição pública e três em instituição privada. Três enfermeiros concluíram a graduação entre 2007 a 2011 e quatro entre 2012 a 2015. Dentre esses, cinco atuam na profissão entre 1 a 3 anos, um entre 3 a 6 anos, 1 entre 6 a 10 anos e um enfermeiro(a) mais de 10 anos. 
Sobre o vínculo empregatício em outra instituição, cinco afirmaram não ter vínculo e três sim. Todos enfermeiros afirmaram não exercer outra função na instituição onde trabalha, e todos afirmaram ter participado de algum curso envolvendo capacitação em obstetrícia. Quanto a carga horária semanal cinco trabalham 24 horas semanais, dois 44 horas semanais, e um, 30 horas semanais.

\section{Categorias Analíticas}

Condutas do enfermeiro com a parturiente no pré-parto

Esta categoria refere-se à observação e aos métodos do cuidado do enfermeiro aplicados na parturiente para atender ao seu estado físico e emocional. Acredita-se que o seu estado físico nesse momento sofre algumas alterações, e é necessário exercícios que irão agilizar e facilitar o parto, assim como o emocional interfere na evolução do parto e pós-parto; o modo como a mulher é cuidada influencia diretamente na forma como ela vivencia esse momento (Porto, Costa \& Velloso, 2015).

Ao questionar os enfermeiros sobre as principais condutas que eles realizam com as gestantes no pré-parto, verificouse que realizam o acolhimento e utilizam métodos estimulantes para a dilatação, com técnicas de relaxamento utilizadas pela instituição:

"[...] chegando aqui a gente acolhe[...] explica direito né!? o preparo do parto, pós-parto, tudo direitinho[...] faz uns exercícios, a gente tem uns equipamentos aqui, a bola suiça, o cavalinho, a escada e também temos um chuveiro né!? A mulher "joga" todas as forças no chuveiro elétrico na água quente, também a gente coloca elas lá pra fazer massagem e pra ajudar né!? nesse processo pra ficar mais tranquila." - ENF 01

“...eu oriento todo o processo de trabalho de parto e vou conduzindo né!? falo das modalidades de posição da livre movimentação, a gente pode utilizar o pélvico com o auxílio da bola suiça, do cavalinho, a gente caminha ao banho de aspersão, se assim ela preferir, ao banho morno dependendo da fase do trabalho de parto, e agachamento..." ENF 04

"Estímulo, deambulação, ausculta de batimentos cardiofetais de uma em uma hora, dinâmica uterina, exames como toque vaginal, monitoramento de pressão arterial, estímulo ao uso de técnicas não farmacológicas para o alivio da dor. a gente tenta ver a questão da respiração, para controlar a respiração, a gente tenta dar apoio emocional." ENF 05

"Admissão das gestantes de risco habitual em fase ativa do trabalho de parto, oferecendo apoio emocional, incentivando a deambulação e verticalização, orientando exercícios respiratórios e de balanço pélvico, e utilizando todas as tecnologias não farmacológicas pra alívio da dor, como massagem, banho em água morna, música e dança, massagem com rebozo, enfim... tentando dessa forma amenizar a dor e tornar um momento marcante de forma positiva na vida daquela mulher[...]." ENF 07

A preparação do parto tem início durante o pré-natal, quando os profissionais de saúde devem realizar a abordagem psicológica do casal e de toda a família (Nomura, Paiva, Costa, Liao \& Zugaib, 2012). Prestar cuidados holísticos atentando para manifestações, por vezes sutis nos comportamentos das mulheres, torna-se uma importante ferramenta para a humanização do cuidado a elas prestado (Ribeiro et al., 2016). 
O cuidado não é apenas a técnica empregada e os métodos assistenciais, envolve a motivação, assim como a gentileza, hostilidade, o respeito e a consideração pelo outro. Por outro lado, há a intencionalidade de promover o bem- estar; de manter o ser seguro e confortável, de oferecer apoio, de minimizar riscos e reduzir a sua vulnerabilidade (Pereira \& Bento, 2011).

As medidas implementadas pelos enfermeiros no acompanhamento humanizado à parturiente têm impacto, pois são cuidados que irão auxiliar no processo natural do parto e são essenciais para nortear o percurso do trabalho de parto.

Os enfermeiros possuem autonomia em realizar assistência em métodos como massagens relaxantes, banhos quentes por aspersão, exercícios com a bola suíça, movimento sob o cavalo, incentivo à deambulação e permanência na posição de cócoras, por períodos suportáveis pelas mulheres-parturientes que estimularão a dilatação (Costa, 2017).

Entre os cuidados relatados, foi observado uma atenção maior para o partograma, por ser um método capaz de acompanhar toda evolução do trabalho de parto e por ser, segundo as falas, um método eficaz.

"[...] a gente usa aqui o partograma pra acompanhar a dilatação, a pressão, os batimentos cardiacos... Tudo é anotado e a gente vai falando pra elas, por exemplo, quando eu recebo uma gestante eu já converso com ela e falo tudo como é o parto [...]” - ENF 02

“[...] a gente procura orientar essa mulher explicando [...] os momentos que ela vai vivenciar. A gente estimula a fazer exercícios

[...] mostra quais são os instrumentos que a gente tem aqui pra acelerar o trabalho de parto [...] e estimula essa mulher a se empoderar... A gente utiliza o instrumento do partograma pra avaliar a evolução desse trabalho de parto, pra isso a gente precisa fazer o toque na mulher e a gente verifica também o BCF." - ENF 03

O partograma é uma representação gráfica do trabalho de parto, permite acompanhar a evolução, dilatação e decida do feto, documentar, diagnosticar caso haja alterações e evitar intervenções desnecessárias (Lima, Leite, Duarte, Fernandes \& Fernandes, 2017). É uma prática que demanda treinamento de baixa complexidade, e que tem grande impacto benéfico auxiliando o enfermeiro obstetra na parturição.

A assistência de qualidade durante o parto mostra a importância do enfermeiro nesse processo. Verificou-se os enfermeiros obstetras respeitam os aspectos da fisiologia feminina sem intervenções desnecessárias, reconhecem os aspectos sociais e culturais do parto e nascimento e oferecem suporte emocional à mulher e a sua família, garantindo os direitos de cidadania.

As tecnologias práticas empregadas durante o pré-parto pelo enfermeiro, como as manobras, estimulação da deambulação, ausência do acompanhante, episiotomia e uso rotineiro de ocitocina provocam divergências entre os profissionais e e atualmente já é considerado violência obstétrica, por não valorizar a autonomia da mulher e respeitar seu processo fisiológico. Para que eventuais não ocorram é de suma importância ter uma equipe capacitada, e sensibilizada com finalidade de que a mulher, seu acompanhante e se bebê sejam respeitados e acolhidos no serviço de forma ética (Gomes et al., 2014).

A Organização Mundial de Saúde preconiza intervenções mínimas possíveis, apenas em casos de insegurança a saúde da parturiente e da criança, sendo então uma razão necessária para interferir sobre o processo natural (Santos \& Monteiro, 2017). Respeitar o momento da mulher, desenvolvendo autonomia para que a parturiente escolha como deve parir contribuirá para que para um melhor desenvolvimento do trabalho de parto. O que para isso é necessário fornecer um bem-estar emocional e físico à mulher, que proporcione a redução de riscos e complicações, com respeito aos direitos da mulher, de modo a proporcionar uma assistência de qualidade (Gomes, et al., 2014). 
"Procuramos respeitar cada parturiente, cada individualidade. Mostramos e oferecemos várias técnicas para alivio da dor e ela escolhe qual a de sua preferência. Ela será a protagonista do seu parto. " ENF 06

"Acolhimento à gestante e ao acompanhante, informando aos mesmos sobre o processo de trabalho de parto, suas fases e da importância da autonomia da mulher nesse processo assim como do acompanhante[...]" ENF 08

A enfermagem obstétrica nesse contexto busca inserir os princípios de cuidado e estimulo a fisiologia do parir, impulsionando o protagonismo feminino, para que esse momento possa ser vivenciado com autonomia das mulheres. Essa autonomia garante a essas pacientes agir de maneira soberana em relação a si mesma (Pereira \& Bento, 2011).

O enfermeiro obstetra deve estar preparado para acolher a gestante, seu companheiro e família, reverenciando todos as etapas e significados que o momento gera nos mesmos. Ao transmitir confiança e tranquilidade, o enfermeiro criará um vínculo o que facilitará as estratégias para humanização da atenção no parto institucionalizado (Gomes et al., 2014).

Os enfermeiros obstetras têm contato direto a parturiente e precisam ter conhecimento suficiente para exercer os cuidados que fornecem humanização, Observou-se, no entanto, que eles têm conhecimento sobre suas ações porém não expõem suas cuidados com detalhes pautados na diferença que o enfermeiro tem ao trabalhar com a gestante nesse momento. Atuam de forma ampla desde a admissão, orientando sobre o processo do trabalho de parto e suas fases, até os cuidados exercidos durante o pré-parto estimulando para que a mesma tenha dilatação rápida e com dor diminuída (Santos et al., 2012).

Foi observado que é necessário, formulação de políticas que expandam as ações realizado pelos enfermeiros obstetras, fazendo com que haja valorização do trabalho, pois ainda há pouco investimento para enfermagem obstétrica, mesmo com a criação da Rede cegonha e com o grande número de instituições que oferecem a especialização em obstetrícia.

\section{Humanização dos enfermeiros obstétricas no cuidado às gestantes}

No processo de humanização da saúde destaca-se o parto. O modelo assistencial traz benefícios físicos e psicológicos a mulher. Parto humanizado tem como significado deixar a mulher agir mantendo controle total de suas ações, participando ativamente do seu próprio cuidado. A equipe atua apenas como facilitadora desse processo (Silva, Nascimento \& Coelho, 2015).

Os enfermeiros relatam como atuam para humanização dos cuidados durante o pré-parto e verificou-se a atenção para o emocional da parturiente, como parte fundamental para ter um trabalho de parto eficaz.

"Eu acho que a gente trabalha tanto com a parte emocional quanto com a fisica[...] pra se empoderar desse trabalho de parto. [...] tentando mostrar que o trabalho de parto não é só o momento, não é só a dor em si[...] tem um motivo pra ela tá passando por toda aquela situação[...]” ENF 01

"[...] a gente trabalha com a parte emocional[...], além de estimular, orientar, a gente procura encorajar ela naquele momento e tentando mostrar que o trabalho de parto não é só a dor que ela tá vivenciando, tem um motivo pra ela estar ali[...] o exercício que ela gosta mais é a bola, e não gostam muito de fazer o agachamento.” ENF 03

$\mathrm{Na}$ fase puerperal as gestantes vivenciam várias situações, durante todo o processo de gestação e parturição. Quando chegam na maternidade trazem consigo toda a vivência, emoções, expectativas. O cuidado prestado pelos enfermeiros, se torna de vital importância, pois com o auxílio, o apoio, a confiança, prestada por estes profissionais, fazem com quer a parturiente seja mais confiante, durante todo o processo do parto (Frigo et al., 2013). 
Os cuidados dos profissionais enfermeiros vão além dos aspectos e procedimentos técnicos. A busca por cuidados que humanizem tanto a parturiente como sua família. Os profissionais de enfermagem ou a equipe de médicos e enfermeiros necessitam desenvolver educação, modos e ações, para executar suas funções como agentes transformadores ou de mudança (Frello, Carraro \& Bernardi, 2011).

O enfermeiro obstetra deve sempre estar em busca de novos conhecimentos, de capacitações na área. Verificou-se que os entrevistados afirmaram que sua vivência na assistência ao parto

"[...] a gente também tem que se colocar no lugar da gestante[...] tem paciente que diz assim: 'ai meu Deus, graças a Deus que tive normal!" [...] a gente estudou tudo que é trabalhado aqui [...] a gente entende que vai trazer beneficios pra ela [...]" - ENF 02

A humanização ao parto e nascimento, o respeito à fisiologia e o resgate ao processo natural da gestação devem ser valorizados como práticas essenciais para o cuidado à mulher. Deve-se buscar o conhecimento baseado em evidências como garantia à segurança a assistência prestada de forma qualificada e digna, proporcionando às mulheres boas práticas obstétricas, livres de riscos e de intervenções que são dispensáveis e que, muitas vezes, ocasionam prejuízos à mulher e a seu recémnascido (Ramos, 2016).

Os enfermeiros têm em sua formação o cuidado humanizado recomendado pela Organização Mundial da Saúde (OMS), onde dará a devida atenção necessária as parturientes, pondo-se sempre que necessário ao dispor e dando a devida atenção específica e qualificada que merecem. O profissional trabalha na construção do cuidado, de forma integrada, promovendo autonomia e incentivos (Mattos, Vandenberghe \& Martins, 2016).

É necessário que os enfermeiros exerçam princípios de humanização da assistência, valorizem o acolhimento, a prática de ouvir a paciente e a relação respeitosa entre profissionais/clientes.

Para colocar o acolhimento em prática, é necessário reorganizar o serviço de saúde a partir da problematização dos processos de produção de trabalho, mudar a forma da estrutura de gestão do serviço de saúde, ter postura de escutar e ter compromisso com os questionamentos trazidos pelas pacientes (Silva, Nascimento \& Coelho, 2015).

"Em geral eu acredito que é o alivio da dor, do conforto, sentir-se bem [...] o respeito à paciente, ela vai se sentir mais acolhida e melhor, enquanto as posições, as tecnologias vão auxiliar pra ter a dilatação e auxiliar na progressão do trabalho de parto." - ENF 04

"a gente dá a garantia do bem-estar[...], melhora a dilatação, evolui mais rápido o trabalho de parto, faz com que o trabalho de parto seja menos doloroso." ENF 05

"os benefícios são: alívio da dor, cria vínculo com a equipe diminuindo medo e ansiedade e a presença do acompanhante sempre ajudando a parturiente." ENF 06

"Conforto, alívio da dor, diminuição da ansiedade, trabalho de parto mais curto e menos intervenções desnecessárias[...] menor números de cesáreas. " ENF 07

Durante o período de trabalho de parto podem haver situações diversas na parturiente, como a dor, a hospitalização, o estado do bebê, que amedrontam a paciente. O enfermeiro entra com orientações, explicações sobre o parto, mantendo- a 
informada. Se a equipe não manter uma relação harmônica com a paciente, a experiência do parto poderá ser traumatizante e poderá haver complicações. Então, para um bom trabalho de parto, é necessário o bem-estar emocional e físico da parturiente (Santos et al., 2012).

Os enfermeiros obstetras que participam no parto, contribuem para a diminuição e eliminação de situações de angustia. Então é por meio da comunicação do enfermeiro que as pacientes adquirem confiança e ficam menos apreensivas. Essa atitude pode proporcionar mudanças no comportamento da gestante. Isso é justificado pela enfermagem conseguir detectar e avaliar o evento doloroso, independentemente do tipo, duração, intensidade, sofrimento e dor de quem o experimenta (Santos \& Monteiro, 2017).

A formação da enfermeira obstetra possibilita habilidades e competência a prestação do cuidado integral a parturiente e sua família, respeitando o parto como um processo fisiológico, fazendo com que a mulher tenha autonomia e escolha como deseja parir.

"[...] envolvem o empoderamento e autonomia das gestantes durante todo o processo de parturição e do vínculo entre mãe e RN[...]. os benefícios envolvem a redução do tempo de trabalho de parto além de auxiliar no alívio da dor durante as contrações. " ENF 08

As parturientes devem ser tratadas com respeito e dignidade, além de terem acesso à informações detalhadas, para isso os profissionais que irão atendê-las devem estar cientes de suas ações, das palavras que irão empregar, do tom de voz e também da forma que esses cuidados serão prestados, todo esse cuidado constitui humanização (Calegari, Massarollo \& Santos, 2017).

$\mathrm{O}$ acolhimento à gestante pelo enfermeiro contribui de forma significativa para um atendimento humanizado, porém essa contribuição deve ser entendida como um processo onde todos estejam qualificados e capacitados para o atendimento. Investir na capacitação é de extrema importância para oferecer uma assistência obstétrica menos invasiva, assumindo a prioridade na redução da morbimortalidade materna e perinatal (Menezes \& Dias, 2012).

É importante considerar as diferenças e semelhanças entre os modelos de assistência e as características próprias de cada local. Os responsáveis pelo atendimento obstétrico necessitam atentar-se para manter uma escuta aproximada da gestante durante todo o processo do pré-parto e ter um olhar diferenciado para elas principalmente na dor, o que a partir das falas dos entrevistados foi possível observar que os enfermeiros atuam diretamente no alívio da dor e métodos que facilitam o trabalho de parto.

\section{Conclusão}

$\mathrm{O}$ estudo possibilitou reconhecer o enfermeiro obstetra como parte de extrema importância dentro da equipe que assiste a parturiente durante o pré-parto. Entender e conhecer os cuidados que ele exerce, é relevante para observar se há ou não humanização. Verificou-se que os cuidados vão desde o acolhimento a gestante e ao acompanhante, o que pode contribuir para um atendimento humanizado, porém isso deve ocorrer quando os profissionais estiverem qualificados e capacitados para o atendimento, até o parto exercendo técnicas holísticas no cuidado da parturiente e seu acompanhante.

Observou-se que os enfermeiros obstetras da maternidade municipal utilizam métodos tecnológicos que facilitam a dilatação pélvica, melhoram a respiração, produzem o relaxamento e ainda aceleram o trabalho de parto. A assistência dos enfermeiros obstetras deve considerar a individualidade de cada mulher e desmitificar que o parto é uma experiência relacionada à dor e ao medo, à dúvida, permitindo as mulheres participem ativamente em todas as etapas do parto como sendo uma experiência dignificante e prazerosa. 
Sabendo que a preparação do parto tem início durante o pré-natal, é imprescindível a puérpera ter um bom relacionamento com a equipe, onde a qual irá acompanhar e assistir o seu parto. Sugere-se então, que haja novos estudos abordando o tema, no intuito de divulgar a importância do enfermeiro obstetra no momento do parto na visão das parturientes, além de desenvolver novas discussões na comunidade acadêmica.

\section{Referências}

Bardin, L. (2016). Análise de Conteúdo. Edições 70.

Calegari, R. D. C., Massarollo, M. C. K. B., \& Santos, M. J. D. (2015). Humanização da assistência à saúde na percepção de enfermeiros e médicos de um hospital privado. Revista da Escola de Enfermagem da USP, 49(SPE2), 42-47.

Costa, R. L. M. (2017). Contribuições da enfermagem obstétrica para uma assistência segura e de qualidade em um hospital público alagoano: um relato de experiência. Gep News, 1(2), 25-31.

Frello, A. T., Carraro, T. E., \& Bernardi, M. C. (2011). Cuidado e conforto no parto: estudos na enfermagem brasileira. Revista Baiana de Enfermagem25 ,(2).

Frigo, J., Ferreira, D. G., Ascari, R. A., Marin, S. M., Adamy, E. K., \& Busnello, G. (2013). Assistência de enfermagem ea perspectiva da mulher no trabalho de parto e parto. Cogitare Enfermagem, 18(4), 761-766.

Gomes, A. R. M., Sousa Pontes, D., Pereira, C. C. A., Brasil, A. D. O. M., \& Moraes, L. D. C. A. (2014). Assistência de enfermagem obstétrica na humanização do parto normal. Revista Recien-Revista Científica de Enfermagem, 4(11), 23-27.

Gomes, M. L., \& Moura, M. A. V. (2012). Modelo humanizado de atenção ao parto no Brasil: evidências na produção científica . Revista Enfermagem UERJ, 20(2), 248-253.

Lima, M. J., Leite, A. R., Duarte, V. F., Fernandes, E. S. B., \& Fernades, N. T. (2017). A utilização do partograma pela enfermagem no trabalho de parto sem distocia. Revista da Universidade Vale do Rio Verde, 15(1), 537-546.

Mattos, D.V., Vandenberghe, L., Martins, C.A. (2016). O enfermeiro obstetra no parto domiciliar planejado. Rev de Enfermagem UFPE on-line. 10(2), p.568575 .

Medeiros, J., Hamad, G. B. N. Z., Costa, R. R. D. O., Chaves, A. E. P., \& Medeiros, S. M. D. (2015). Métodos não farmacológicos no alívio da dor de parto: percepção de puérperas. Espaç Saúde [Internet], 16(2), 37-44.

Menezes, M. G. B., \& Dias, D. F. S. (2012). A humanização do cuidado no pré-parto e parto. SynThesis Rev Digital FAPAM. 3(3),24-36.

Nomura, R. M. Y., Paiva, L. V., Costa, V. N., Liao, A. W., \& Zugaib, M. (2012). Influência do estado nutricional materno, ganho de peso e consumo energético sobre o crescimento fetal, em gestações de alto risco. Revista Brasileira de Ginecologia e Obstetrícia, 34(3), 107-112.

Oliveira, E. C., Meira Barbosa, S., \& Melo, S. E. P. (2016). A importância do acompanhamento pré-natal realizado por enfermeiros. Revista Científica FacMais, 7(3).

Pereira, A. L.F., \& Bento, A. D. (2011). Autonomia no parto normal na perspectiva das mulheres atendidas na casa de parto. Rev Rene, 12 (3), $471-477$.

Pieszak, G. M., Terra, M. G., Neves, E. T., Pimenta, L. F., Mello Padoin, S. M., \& Ressel, L. B. (2013). Percepção dos profissionais de enfermagem acerca do cuidar em centro obstétrico. Revista da Rede de Enfermagem do Nordeste, 14(3), 568-578.

Porto, A. A. S., da Costa, L. P., \& Velloso, N. A. (2015). Humanização da assistência ao parto natural: uma revisão inte grativa. Ciência \& Tecnologia, 1(1), 12-19.

Ramos, V. M. A. (2016) Assistência da enfermeira obstétrica ao parto baseado em evidências. 88 f. Dissertação (Mestrado em Enfermagem) - Universidade Federal do Estado do Rio de Janeiro, Rio de Janeiro.

Rezende, M. M. R. D., Soares, N. R., Silva, R. C. V. (2015). Atuação humanizada no parto natural sob a ótica da enfermeira obstetra. 11 f. Trabalho de Conclusão de Curso (Graduação em Enfermagem) - Universidade Federal da Bahia.

Ribeiro, J. F., Machado, P. H. F., Silva Araújo, K. R., \& Abreu Sepúlvedra, B. (2016). Assistência ao parto normal sob o olhar da parturiente. Revista Eletrônica Gestão e Saúde, (1), 113-125.

Santos, A. C., Monteiro, R. A. L. S. (2017). Métodos não farmacológicos utilizados pelo enfermeiro para alivio da dor no trabalho de parto. Rev Nambiquara, $6(1), 77-86$.

Santos, G. S., Souza, J. L., Almeida, L. S. D., \& Gusmão, M. H. (2012). A importância do enfermeiro no atendimento humanizado no pré-parto. Diálogos Ciênc [Internet], 224-8.

Silva, A. L. S., Nascimento, E. R. D., \& Coelho, E. D. A. C. (2015). Práticas de enfermeiras para promoção da dignificação, participação e autonomia de mulheres no parto normal. Escola Anna Nery, 19(3), 424-431. 